\title{
Luminal matrices: An inside view on organ morphogenesis
}

\author{
Luschnig, Stefan ; Uv, Anne
}

\begin{abstract}
Tubular epithelia come in various shapes and sizes to accommodate the specific needs for transport, excretion and absorption in multicellular organisms. The intestinal tract, glandular organs and conduits for liquids and gases are all lined by a continuous layer of epithelial cells, which form the boundary of the luminal space. Defects in epithelial architecture and lumen dimensions will impair transport and can lead to serious organ malfunctions. Not surprisingly, multiple cellular and molecular mechanisms participate in controlling size and form of tubular epithelial structures. One intriguing aspect of epithelial organ formation is the coordinate behavior of individual cells as they mold the mature lumen. Here, we focus on recent findings, primarily from Drosophila, demonstrating that informative cues can emanate from the developing organ lumen in the form of solid luminal material. The luminal material is produced by the surrounding epithelium and helps to coordinate changes in shape and arrangement of the very same cells, resulting in correct lumen dimensions.
\end{abstract}

DOI: https://doi.org/10.1016/j.yexcr.2013.09.010

Posted at the Zurich Open Repository and Archive, University of Zurich

ZORA URL: https://doi.org/10.5167/uzh-81590

Journal Article

Accepted Version

Originally published at:

Luschnig, Stefan; Uv, Anne (2014). Luminal matrices: An inside view on organ morphogenesis. Experimental Cell Research, 321(1):64-70.

DOI: https://doi.org/10.1016/j.yexcr.2013.09.010 


\title{
Luminal matrices: An inside view on organ morphogenesis
}

\author{
Stefan Luschnig ${ }^{1 *}$ and Anne $\mathrm{Uv}^{2 *}$ \\ ${ }^{1}$ Institute of Molecular Life Sciences, University of Zurich, Winterthurerstrasse 190, \\ CH-8057 Zurich, Switzerland \\ ${ }^{2}$ Department of Medical Genetics, Institute of Biomedicine, Sahlgrenska Academy, \\ University of Gothenburg, SE-40530 Gothenburg, Sweden
}

*Correspondence: anne.uv@gu.se and stefan.luschnig@imls.uzh.ch

Keywords: apical extracellular matrix, organogenesis, tubulogenesis, lumen, chitin, mechanical forces, luminal pressure 


\begin{abstract}
Tubular epithelia come in various shapes and sizes to accommodate the specific needs for transport, excretion and absorption in multicellular organisms. The intestinal tract, glandular organs and conduits for liquids and gases are all lined by a continuous layer of epithelial cells, which form the boundary of the luminal space. Defects in epithelial architecture and lumen dimensions will impair transport and can lead to serious organ malfunctions. Not surprisingly, multiple cellular and molecular mechanisms participate in controlling size and form of tubular epithelial structures. One intriguing aspect of epithelial organ formation is the coordinate behaviour of individual cells as they mould the mature lumen. Here, we focus on recent findings, primarily from Drosophila, demonstrating that informative cues can emanate from the developing organ lumen in the form of solid luminal material. The luminal material is produced by the surrounding epithelium and helps to coordinate changes in shape and arrangement of the very same cells, resulting in correct lumen dimensions.
\end{abstract}




\section{Introduction}

Many developing epithelial organs start out as small tubular primordia that will expand to give rise to mature functional organs. The cells constituting these organ primordia are polarized along the apical-basal axis and connect to each other via junctional complexes that maintain polarity and provide adhesive functions. While the apical plasma membrane generally faces the lumen and exhibits specialized secretory functions, the basal membrane rests on a surrounding basal lamina. Additional cell layers, often of mesodermal origin, may encircle the tubular epithelium to provide support and contractile forces to the organ. The subsequent size-maturation of primordial organ lumina involves distinct and preprogramed phases of growth, both in length and diameter, to attain dimensions optimal for organ function. Such tube growth is mediated by highly coordinated changes in cell shape and cell rearrangements, and it can be accompanied by cell proliferation as the entire organ grows.

Key cellular processes involved in epithelial lumen morphogenesis include membrane and protein trafficking, cytoskeletal changes, junction rearrangement, growth and cell division. For instance, the addition of apical membrane to accommodate rapid lumen diameter expansion can be driven by exocytosis, as in the Drosophila tracheae $[1,2]$, or by reshuffling of intracellular membrane to the apical surface, as in the excretory organ in C. elegans [3]. Oriented cell intercalation, cell division and cell elongation also participate in regulating lumen dimensions during size-maturation of the organ. In the developing Drosophila tracheae, axial tube elongation relies on polarized cell shape changes along the tube axis $[4,5]$, while in Xenopus embryos, the pronephric tubules elongate through rosette-based cell intercalation [6]. The latter is similar to the highly stereotyped cell intercalation 
events observed during germband elongation in Drosophila [7], and both processes depend on planar cell polarity (PCP) signalling. PCP appears to control the spatially oriented cell rearrangements and the orientation of cell divisions along the renal tube axis, and defects in this process have been proposed to cause cyst formation in polycystic kidney disease (PKD) [8]. The stability and proper dimensions of lumina in epithelial tubes are further supported by subapical actin and the intermediate filamentbased terminal web $[9,10]$.

An interesting problem is how the diverse cellular events are coordinated across the epithelium to produce a correctly shaped lumen. Part of the answer may rely on the transmission of forces within the tissue to produce coordinate global changes in shape. Over the past years, much progress has been made in describing tissue and organ morphogenesis as biomechanical processes [11]. The self-organizing features of actin networks, the dynamics of actomyosin contractility, and the dynamic remodelling of cell-cell junctions and ECM all contribute to the mechanical properties of epithelial tissues. Tubular epithelia present a special case with regard to tissue mechanics, as they form lumina that can provide an additional source for force generation and integration to steer cellular behaviour in the surrounding epithelium. Luminal hydrostatic pressure, for example, generated by liquid secretion or osmosis, will exert a uniform force on the enclosing cells (Fig. 1A and 1B). Indeed, such hydrostatic pressure was proposed to play important roles in lumen formation and expansion in the gut [12], in Kupffer's vesicle [13] and in brain ventricles [14] in zebrafish, and in the excretory cell [15] and vulva [16] in C. elegans. Moreover, the cylindrical geometry of tubular structures brings along physical constraints that are distinct from those experienced by cells in planar epithelial sheets. Laplace's law states that circumferential surface tension on a pressurized cylinder is larger than axial 
surface tension. This force anisotropy can be exemplified by an over-boiled sausage, which always bursts along its length (Fig. 1A). Thus, although the distribution and scale of forces in tubular epithelia have not been well documented, luminal pressure can in principle also generate planar asymmetry along the tube axis. Aside from hydrostatic pressure, flow of the luminal content can generate shear stress that acts on cells lining the apical surface of the tube wall. In the vascular and lymphatic systems, such sheer stress activates mechanosensory signalling and leads to various cellular effects, including changes in gene expression [17].

A recently discovered way of utilizing the lumen as a source of cues to organize remodelling of the surrounding cells depends on solid luminal material. Such luminal matrices are transiently deposited by the expanding epithelium and have important roles in determining the size and shape of the organ, possibly by generating and integrating mechanical forces across the luminal space. In the following, we discuss functional aspects of such luminal matrices during Drosophila tubular organ development, and consider the occurrence, molecular composition, and functions of luminal components in various model systems. By integrating findings obtained in Drosophila and in vertebrates, we highlight the potential general relevance of the luminal matrix concept for diverse types of organs and organisms.

\section{Drosophila tracheal tubes are shaped by a luminal chitin matrix}

Maturation of epithelial lumen size has been extensively studied in the Drosophila tracheal system, a branched tubular network built from a simple singlelayered epithelium. The two main tracheal tubes (dorsal trunks), with two to five cells surrounding the lumen circumference (Fig. 2A), are initially formed as straight narrow tubes running along the anterior to posterior axis of the animal. In the absence 
of further cell division, they undergo discrete growth phases leading to mature diameter and length [18]. Diameter expansion involves substantial and rapid growth of the luminal surface, whereas the outer tube diameter remains fairly constant (Fig. 2A). During this period, new apical membrane is added through apical secretion, and animals with impaired secretion fail to expand the tracheal lumen while completing many other morphogenetic processes $[1,2]$. Tracheal tube elongation is controlled independently of diametric expansion and requires the tyrosine kinase Src42 for polarized cell shape changes in the axial dimension of the tubes $[4,5]$. Src42 could be involved in sensing anisotropic mechanical forces or in translating such mechanical cues into oriented cell elongation by regulating adherens junction remodelling [4] and actin polymerization through effects on the formin DAAM [5].

In case of the embryonic tracheal tubes, diametric growth of the lumen is driven by secretion to increase the apical cell surface area [1]. Yet, genes involved in chitin synthesis and chitin organization are essential for uniform diameter expansion. Chitin, a polymer of beta-1,4-N-Acetylglucosamine (GlcNAc), is produced by transmembrane chitin synthases that convert cytoplasmic UDP-GlcNAc to long polysaccharide chains that extrude into the apical extracellular space. Just prior to diameter expansion, the tracheal cells begin to deposit chitin to form a transient fibrelike filament that fills the luminal space during diameter growth (Fig. 2A). Strikingly, in mutant animals that lack tracheal chitin, the tracheal lumen develops severe local dilations and constrictions along the entire tube length. (Fig. 2A) [19, 20]. Inhibition of chitin synthesis, or expression of an apically secreted chitinase, produces similar effects, arguing that the intraluminal chitin functions to shape the lumen.

An important feature of chitin is the ability to form matrices with different physical properties. Nascent chitin chains form microfibrils of about 20 chains that 
can organize into different types of structures depending on associated proteins [21]. Correct organization of chitin in the tracheal lumen is essential for its function, since defects in chitin structure, as observed in mutants for knickkopf $(k n k)$ or retroactive $(r t v)$, also lead to an uneven lumen diameter, similar to what is seen upon loss of chitin $[19,22]$. Knk and Rtv are secreted membrane-bound proteins required for correct chitin organization both in the tracheal lumen and later in the apical cuticle. The chitin matrix therefore appears to provide a physical scaffold to shape the organ, consistent with the tube defects upon fragmentation of chitin chains [20]. The matrix conceivably attaches to the apical surface of surrounding tracheal cells, thereby acting to simultaneously push and hold back the tube wall and mould the lumen into a shape determined by the form and rigidity of the matrix itself (Fig. 1B). Despite intensive mutagenesis screens to detect new tracheal tube size genes, there is no report yet of a candidate molecule that can mediate attachment between the chitinous matrix and the apical cell surface. However, chitin synthases are processive enzymes that remain bound to the growing polymer through multiple polymerization steps, and, in analogy with cellulose synthases, it is possible that chitin synthase subunits form supramolecular complexes, allowing the nascent chitin chains to spontaneously form microfilaments [21]. Such microfilaments, being attached to the chitin synthases at one end and incorporated into the chitinous matrix at the other, could effectively serve as anchors.

Proteins embedded in chitinous matrixes can alter the structure of the matrix and modulate its stiffness, elasticity and accessibility to degradation [21]. Furthermore, chitin fibrils can be enzymatically modified by deacetylation, which alters the chemical and mechanical properties of the chitin polymer. Two secreted chitin deacetylases, Serpentine (Serp) and Vermiform (Verm), contain chitin binding 
and chitin deacetylase domains [23, 24]. Mutations in these genes affect the structure of both the cuticular and tracheal chitinous matrices. Intriguingly, tracheal tube diameter is normal in animals that lack either protein, but the tubes grow excessively in length. The over-elongated tracheal tubes in serp and verm mutants suggest that the chitin matrix acts to limit tube elongation, and that the proteins affect the longitudinal tensional strength of the matrix. Recently, two other proteins with chitin-binding domains, Obst-A and Gasp, were shown to be required for full diameter expansion of the tracheal lumen $[25,26]$. Given that the proteins affect the assembly of chitinous matrices, the proteins might increase the diametric tensional force of the luminal matrix needed for full diametric lumen expansion.

To summarize, research on tracheal tube maturation shows that cell intrinsic mechanisms, such as apical secretion and oriented cell elongation, are prerequisites for lumen growth, and that luminal chitin matrices provide essential physical constraints serving to coordinate changes in cell shape to adjust both tube diameter and length.

\section{A non-chitinous matrix drives diameter expansion of the Drosophila hindgut}

While a main component of the tracheal luminal matrix is chitin, vertebrates do not synthesize chitin. Also in Drosophila, intraluminal chitin is detected exclusively in the developing tracheal network. Thus, a simple extrapolation of the findings from the Drosophila trachea is not possible based on molecular homologues. However, it was recently shown that the large luminal glycoprotein Tenectin (Tnc) forms a luminal matrix that acts to drive tube diameter expansion in the developing Drosophila hindgut [27]. The Tnc matrix spans the lumen and causes dose-dependent expansion, suggesting that it generates a luminal physical force during tube growth. 
The embryonic hindgut consists of about 700 epithelial cells surrounded by visceral muscle cells. After formation of the primordial hindgut tube, which is shaped like the shaft of an umbrella, the tube grows in length and diameter over a three-hour period to attain its final dimensions (Fig. 2B). Lumen diameter expansion is not uniform along the tube axis, but the "crook handle" of the umbrella shaft (small intestine) expands to a larger extent than the straight part (large intestine). As no cell division takes place during lumen growth, the process is mediated by increase in cell size, flattening of the cells along the apico-basal axis and rearrangement of cells [28]. While Tnc forms a continuous fibrillar material in the entire hindgut lumen, Tnc expression levels are higher in the small intestine than in the large intestine (Fig. 2B) $[27,29]$. In tnc mutants, the hindgut tube diameter remains narrow, while overexpression of Tnc leads to diametric overexpansion in a dose-dependent fashion (Fig. 1B). At the cellular level, Tnc promotes an increase in apical cell surface, oriented cell elongation along the tube perimeter and cell intercalation to yield more cells along the lumen perimeter [27]. These effects are similar to those caused by hydrostatic pressure during inflation of the zebrafish brain ventricle [14], expansion of the mouse blastocyst [30], and growth of renal cysts in vitro [31, 32]. Given that Tnc contains two large mucin-domains rich in serine and threonine that are substrates for O-glycosylation, it is possible that hydration of Tnc in the lumen mediates the formation of a gel-like matrix that can exert a distending force on the surrounding tube wall. Consistent with this idea, Tnc exerts its function as a secreted protein within the lumen, and it is able to cause abnormal tube diameter when misexpressed in other tubular organs. Moreover, at sites of Tnc secretion, the protein forms a lumen-spanning complex with low mobility, causing local dilation. It therefore seems 
that Tnc can cause regional effects on diameter expansion dependent on its level of expression along the tube axis [27].

Although both Tnc- and chitin-containing luminal matrices are important to shape epithelial structures, the two matrices exert different functions during tube diameter expansion. The former drives tube diameter expansion in a dose-dependent manner that can be explained by an increased intraluminal pressure, whereas the chitin matrix does not promote expansion, but rather maintains a uniform lumen during secretion-driven diameter expansion. In this instance, specific attachment of chitin to the surrounding epithelium is likely to be involved (Fig. 1B).

\section{Additional luminal cues in epithelial organ morphogenesis}

Large glycoproteins are common among species, and there is evidence that glycosylated components are transiently present in the lumina of different types of developing epithelial organs. The application of fluorescently conjugated lectins to detect various O-linked and N-linked glycans in Drosophila embryos revealed staining in the lumina of the salivary glands, fore- and hindgut, and the tracheae [33]. Moreover, an antibody against the Tn antigen (GalNAc $\alpha-$ Ser/Thr) that detects Oglycans, stained the lumina of the same organs [33]. Luminal lectin staining in epithelial organs during lumen growth is not restricted to the fly embryo, but has also been documented for the embryonic kidney [34] and lung [35] in vertebrates. Notably, during the initial formation of parabronchi and atria in chick embryos, various lectins stained the surface and cytoplasmic granules of the lining epithelial cells, but in subsequent phases, the parabronchial lumen and the atrial cavities were characterized by the presence of lectin-reactive material [35]. This lectin-reactive material disappeared a few days before birth, which would be consistent with a function during 
lumen growth. In the same way, an electron-dense material is detectable in the embryonic C. elegans excretory cell lumen after formation of the luminal surface. The matrix is present during lumen elongation and ramification, and disappears before hatching, when intracellular electron-dense material appears in the subapical cortex [36]. One component of the excretory cell luminal matrix is the mucin-like protein let-653 [36, 37]. In let-653 mutants the worm's single-celled excretory canals swell into large cysts. Although the molecular mechanism is not known, it is conceivable that the let-653 matrix and the luminal chitin filament in the Drosophila trachea provide similar functions in modelling or stabilizing luminal shape.

In Drosophila, Tnc is found in the developing lumina of several organs, but it appears that Tnc contributes to detectable luminal O-glycans only in the hindgut. Thus, additional luminal O-glycosylated proteins are probably abundant in other organ lumina. These might correspond to a subset of mucin-like proteins that are dynamically expressed in embryonic epithelial organs during size maturation [38], and to other unknown glycoproteins. Luminal proteins might also contribute to shaping the lumen of the salivary gland in Drosophila [39]. Ultrastructural analysis of the wild-type salivary gland shows that the apical cell surfaces surround a relatively large luminal space filled with a fibrillar matrix. Embryos that lack SG1 and SG2, two subunits of ER-resident prolyl 4-hydroxylase that hydroxylates prolines in secreted and transmembrane proteins, show a reduced volume and altered structure of salivary gland secretions. These altered secretions are associated with regions of tube dilation and constriction and intermittent tube closures, suggesting that modification of luminal proteins by SG1 and SG2 is required for formation of an expanded fibrillar matrix to maintain an open and uniform tube [39]. Moreover, two secreted Zona Pellucida (ZP) proteins, Piopio (Pio) and Dumpy (Dp), are deposited in the tracheal 
lumen and are essential for maintaining integrity of the tubes during tracheal cell intercalation [40]. Interestingly, Pio and $\mathrm{Dp}$, a giant protein of $2.5 \mathrm{MDa}$, are also required for maintaining stable attachment between epithelial sheets in the fly wings and for epidermal-cuticle attachment, suggesting a general function of these proteins in determining mechanical properties of epithelia.

Apart from components of the aforementioned luminal matrices, luminal glycoproteins can facilitate the separation of apical membranes during lumen formation. It has been proposed that membrane detachment might involve steric hindrance of cell-cell adhesion by large transmembrane glycoproteins [41]. Indeed, the initial separation of apical membranes during aortic tube development in the mouse requires the apical sialomucin Podocalyxin [42], whose negatively charged sialic acid moieties are thought to cause electrostatic repulsion of the apical surfaces. In the Drosophila retina, a large predicted proteoglycan called Eyes Shut (Eys) [43] is apically secreted by the photoreceptor cells that form a neuroepithelium. Clusters of photoreceptor cells enclose with their apical surfaces a channel-like extracellular space called the interrhabdomeric space, into which Eys is deposited. Eys is required to separate the apical membranes of the photoreceptors to form the interrhabdomeric space, but its exact mechanism of action is not known.

Along with continued research to reveal new protein functions in epithelial organ morphogenesis, the list of luminal components with roles in epithelial remodelling will likely expand. Their functional characterization is promising to reveal further insights into how organ lumina contribute chemical and mechanical cues during tissue remodelling. 


\section{Perspectives}

Apical extracellular matrices have previously been described mainly in the context of their roles in organ physiology, protecting the epidermis and internal epithelial surface from physical and chemical damage, dehydration and infection. With this review we highlight important functions of apical ECMs in organ morphogenesis, which have become evident only over the past decade. A key concept is that the luminal material provides a global cue to the epithelium and is thereby capable of coordinating cell behaviour over long distances within a particular organ. Recent work has shown that luminal matrices can be built from different types of macromolecules, such as long polysaccharides or large glycoproteins, and that different types of matrices have different properties and effects on the surrounding epithelium. Unlike hydrostatic pressure, which is isotropic, solid luminal matrices can exert differential forces along the tube axis, and these forces can be generated also at developmental stages when epithelial paracellular barrier functions (which are essential to generate luminal hydrostatic pressure) are not yet established. A luminal matrix can also act as a scaffold for the surrounding cells and, through attachments to the apical surface of the tube wall, impose a restraint on diametric expansion and tug or hold back tube elongation (Fig. 1B). Such force integration along the luminal matrix may facilitate the coordination of cell behaviour across the epithelium. It currently remains largely unexplored how cells sense and respond to the mechanical cues from luminal matrices. The deformation (contraction, stretching) imposed by the luminal matrix will plausibly challenge load-carrying adhesions and structural elements beyond the elasticity range of the tissue, resulting in plastic tissues changes. Further studies will be required to assess the scale and distribution of mechanical forces within organ lumina, and to elucidate which cellular elements mediate 
resistance and adaptations to these forces. This will involve characterizing and manipulating the level of stretching, compression or relaxation that is imposed by luminal matrices, as well as studying the biological mechanisms of how mechanical cues are translated into intracellular changes.

\section{Acknowledgments}

We would like to apologize to colleagues whose work could not be cited due to space limitations.

Research in AU's laboratory is supported by the Swedish Research Council (VR-M) and the Swedish Cancer Society. Research in SL's laboratory is supported by the Swiss National Science Foundation (SNF 31003A_141093_1), the University of Zürich, and the Kanton Zürich.

\section{References}

[1] D. Förster, K. Armbruster, S. Luschnig, Sec24-dependent secretion drives cell-autonomous expansion of tracheal tubes in Drosophila, Curr Biol 20 (2010) 62-68.

[2] V. Tsarouhas, K. Senti, S. Jayaram, K. Tiklová, J. Hemphälä, J. Adler, C. Samakovlis, Sequential pulses of apical epithelial secretion and endocytosis drive airway maturation in Drosophila, Developmental cell 13 (2007) 214-225.

[3] I. Kolotuev, V. Hyenne, Y. Schwab, D. Rodriguez, M. Labouesse, A pathway for unicellular tube extension depending on the lymphatic vessel determinant Prox1 and on osmoregulation, Nature cell biology 15 (2013) 157-168. 
[4] D. Förster, S. Luschnig, Src42A-dependent polarized cell shape changes mediate epithelial tube elongation in Drosophila, Nature cell biology 14 (2012) 526-534.

[5] K.S. Nelson, Z. Khan, I. Molnar, J. Mihaly, M. Kaschube, G.J. Beitel, Drosophila Src regulates anisotropic apical surface growth to control epithelial tube size, Nature cell biology 14 (2012) 518-525.

[6] S.S. Lienkamp, K. Liu, C.M. Karner, T.J. Carroll, O. Ronneberger, J.B. Wallingford, G. Walz, Vertebrate kidney tubules elongate using a planar cell polarity-dependent, rosette-based mechanism of convergent extension, Nature genetics 44 (2012) 1382-1387.

[7] J.T. Blankenship, S.T. Backovic, J.S. Sanny, O. Weitz, J.A. Zallen, Multicellular rosette formation links planar cell polarity to tissue morphogenesis, Developmental cell 11 (2006) 459-470.

[8] E. Fischer, E. Legue, A. Doyen, F. Nato, J.F. Nicolas, V. Torres, M. Yaniv, M. Pontoglio, Defective planar cell polarity in polycystic kidney disease, Nature genetics 38 (2006) 21-23.

[9] V. Gobel, P.L. Barrett, D.H. Hall, J.T. Fleming, Lumen morphogenesis in C. elegans requires the membrane-cytoskeleton linker erm-1, Developmental cell 6 (2004) 865-873.

[10] K. Carberry, T. Wiesenfahrt, F. Geisler, S. Stocker, H. Gerhardus, D. Uberbach, W. Davis, E. Jorgensen, R.E. Leube, O. Bossinger, The novel intestinal filament organizer IFO-1 contributes to epithelial integrity in concert with ERM-1 and DLG-1, Development (Cambridge, England) 139 (2012) 1851-1862.

[11] T. Lecuit, P.F. Lenne, E. Munro, Force generation, transmission, and integration during cell and tissue morphogenesis, Annual review of cell and developmental biology 27 (2011) 157-184.

[12] M. Bagnat, I.D. Cheung, K. Mostov, D.Y. Stainier, Genetic control of single lumen formation in the zebrafish gut, Nat Cell Biol 9 (2007) 954-960. 
[13] A. Navis, L. Marjoram, M. Bagnat, Cftr controls lumen expansion and function of Kupffer's vesicle in zebrafish, Development (Cambridge, England) 140 (2013) 1703-1712.

[14] J. Zhang, J. Piontek, H. Wolburg, C. Piehl, M. Liss, C. Otten, A. Christ, T.E. Willnow, I.E. Blasig, S. Abdelilah-Seyfried, Establishment of a neuroepithelial barrier by Claudin5a is essential for zebrafish brain ventricular lumen expansion, Proceedings of the National Academy of Sciences of the United States of America 107 (2010) 1425-1430.

[15] L.A. Khan, H. Zhang, N. Abraham, L. Sun, J.T. Fleming, M. Buechner, D.H. Hall, V. Gobel, Intracellular lumen extension requires ERM-1-dependent apical membrane expansion and AQP-8-mediated flux, Nature cell biology 15 (2013) 143-156.

[16] S. Farooqui, M.W. Pellegrino, I. Rimann, M.K. Morf, L. Muller, E. Frohli, A. Hajnal, Coordinated lumen contraction and expansion during vulval tube morphogenesis in Caenorhabditis elegans, Developmental cell 23 (2012) 494506.

[17] B.L. Roman, K. Pekkan, Mechanotransduction in embryonic vascular development, Biomech Model Mechanobiol 11 (2012) 1149-1168.

[18] G. Beitel, M. Krasnow, Genetic control of epithelial tube size in the Drosophila tracheal system, Development (Cambridge, England) 127 (2000) 3271-3282.

[19] W.P. Devine, B. Lubarsky, K. Shaw, S. Luschnig, L. Messina, M. Krasnow, Requirement for chitin biosynthesis in epithelial tube morphogenesis, Proc Natl Acad Sci USA 102 (2005) 17014-17019.

[20] A. Tonning, J. Hemphälä, E. Tång, U. Nannmark, C. Samakovlis, A. Uv, A transient luminal chitinous matrix is required to model epithelial tube diameter in the Drosophila trachea, Developmental cell 9 (2005) 423-430.

[21] H. Merzendorfer, Insect chitin synthases: a review, J Comp Physiol B 176 (2006) 1-15.

[22] B. Moussian, E. Tang, A. Tonning, S. Helms, H. Schwarz, C. NussleinVolhard, A.E. Uv, Drosophila Knickkopf and Retroactive are needed for 
epithelial tube growth and cuticle differentiation through their specific requirement for chitin filament organization, Development (Cambridge, England) 133 (2006) 163-171.

[23] S. Luschnig, T. Bätz, K. Armbruster, M. Krasnow, serpentine and vermiform encode matrix proteins with chitin binding and deacetylation domains that limit tracheal tube length in Drosophila, Curr Biol 16 (2006) 186-194.

[24] S. Wang, S. Jayaram, J. Hemphälä, K. Senti, V. Tsarouhas, H. Jin, C. Samakovlis, Septate-junction-dependent luminal deposition of chitin deacetylases restricts tube elongation in the Drosophila trachea, Curr Biol 16 (2006) 180-185.

[25] K. Tiklova, V. Tsarouhas, C. Samakovlis, Control of airway tube diameter and integrity by secreted chitin-binding proteins in Drosophila, PLoS ONE 8 (2013) e67415.

[26] G. Petkau, C. Wingen, L.C. Jussen, T. Radtke, M. Behr, Obstructor-A is required for epithelial extracellular matrix dynamics, exoskeleton function, and tubulogenesis, The Journal of biological chemistry 287 (2012) 2139621405 .

[27] Z.A. Syed, A.L. Bouge, S. Byri, T.M. Chavoshi, E. Tang, H. Bouhin, I.F. van Dijk-Hard, A. Uv, A luminal glycoprotein drives dose-dependent diameter expansion of the Drosophila melanogaster hindgut tube, PLoS genetics 8 (2012) e1002850.

[28] D.D. Iwaki, K.A. Johansen, J.B. Singer, J.A. Lengyel, drumstick, bowl, and lines are required for patterning and cell rearrangement in the Drosophila embryonic hindgut, Developmental biology 240 (2001) 611-626.

[29] S. Fraichard, A.L. Bouge, I. Chauvel, H. Bouhin, Tenectin, a novel extracellular matrix protein expressed during Drosophila melanogaster embryonic development, Gene Expr Patterns 6 (2006) 772-776.

[30] A.J. Watson, D.R. Natale, L.C. Barcroft, Molecular regulation of blastocyst formation, Anim Reprod Sci 82-83 (2004) 583-592.

[31] A. Ferrari, A. Veligodskiy, U. Berge, M.S. Lucas, R. Kroschewski, ROCKmediated contractility, tight junctions and channels contribute to the 
conversion of a preapical patch into apical surface during isochoric lumen initiation, Journal of cell science 121 (2008) 3649-3663.

[32] H. Li, I.A. Findlay, D.N. Sheppard, The relationship between cell proliferation, Cl- secretion, and renal cyst growth: a study using CFTR inhibitors, Kidney Int 66 (2004) 1926-1938.

[33] E. Tian, K.G. Ten Hagen, O-linked glycan expression during Drosophila development, Glycobiology 17 (2007) 820-827.

[34] K. Schumacher, R. Strehl, W.W. Minuth, Detection of glycosylated sites in embryonic rabbit kidney by lectin chemistry, Histochem Cell Biol 118 (2002) 79-87.

[35] G. Gheri, E. Sgambati, S.G. Bryk, Glycoconjugate sugar residues in the chick embryo developing lung: a lectin histochemical study, J Morphol 243 (2000) 257-264.

[36] M. Buechner, Tubes and the single C. elegans excretory cell, Trends in cell biology 12 (2002) 479-484.

[37] S.J. Jones, D.L. Baillie, Characterization of the let-653 gene in Caenorhabditis elegans, Molecular \& general genetics : MGG 248 (1995) 719-726.

[38] Z.A. Syed, T. Hard, A. Uv, I.F. van Dijk-Hard, A potential role for Drosophila mucins in development and physiology, PLoS ONE 3 (2008) e3041.

[39] E.W. Abrams, W.K. Mihoulides, D.J. Andrew, Fork head and Sage maintain a uniform and patent salivary gland lumen through regulation of two downstream target genes, PH4alphaSG1 and PH4alphaSG2, Development (Cambridge, England) 133 (2006) 3517-3527.

[40] A. Jaźwińska, C. Ribeiro, M. Affolter, Epithelial tube morphogenesis during Drosophila tracheal development requires Piopio, a luminal ZP protein, Nat Cell Biol 5 (2003) 895-901.

[41] L.E. O'Brien, M.M. Zegers, K. Mostov, Opinion: Building epithelial architecture: insights from three-dimensional culture models, Nat Rev Mol Cell Biol 3 (2002) 531-537. 
[42] B. Strilic, J. Eglinger, M. Krieg, M. Zeeb, J. Axnick, P. Babal, D.J. Muller, E. Lammert, Electrostatic cell-surface repulsion initiates lumen formation in developing blood vessels, Curr Biol 20 (2010) 2003-2009.

[43] N. Husain, M. Pellikka, H. Hong, T. Klimentova, K.M. Choe, T.R. Clandinin, U. Tepass, The agrin/perlecan-related protein eyes shut is essential for epithelial lumen formation in the Drosophila retina, Developmental cell 11 (2006) 483-493. 


\section{Figure legends}

\section{Figure 1. Physical cues in epithelial tube expansion}

A) Internal isotropic pressure in a cylinder will produce hoop stress $\left(\sigma_{H}\right)$ and longitudinal stress $\left(\sigma_{\mathrm{L}}\right)$ in the tube wall. The hoop stress is larger than the longitudinal stress, as illustrated by the longitudinally ruptured skin of an over-boiled sausage.

B) Three ways that physical luminal cues can act during diameter expansion of a tubular primordium. Left: Hydrostatic pressure generates equal force normal to the lumen surface. Depending on the magnitude and duration of force and on the mechanical properties of the tissue, the resulting deformation can lead to expansion of the entire tube diameter. Middle: Apical membrane growth results in lumen dilation. A rigid luminal matrix serves as a scaffold that holds on to the lumen surface and supports uniform lumen diameter growth. Right: A luminal matrix generates an internal pressure. The low mobility of the matrix inside the lumen facilitates differential lumen dilation along the tube axis, depending on the local amount of matrix deposition.

Figure 2. Roles for luminal matrices in diametric expansion of the Drosophila tracheae and hindgut.

A) Top, left: The lumen of tracheal tubes grows by apical membrane growth and cell flattening. The apical surface is drawn in magenta and the luminal matrix in green. Top, middle: Transmission electron microscopy reveals the secretory activity of tracheal cells early during expansion (top panel) and the presence of solid material in the lumen centre during dilation. Top, right: Live imaging of tracheal cells expressing cytoplasmic GFP (blue) and the luminal chitin-binding protein, Verm::RFP (green) 
shows how the luminal component fills the lumen during expansion. Bottom: The luminal chitin matrix, detected by a fluorescently conjugated chitin-binding protein (green), forms a filamentous matrix inside the lumen, which is delineated by labelling for the apical protein, Crumbs (Crb; magenta). In $k k v$ mutants, chitin is missing and the tubes develop local dilations and constrictions.

B) Top: The lumen of the small ( $\mathrm{Si}$ ) and large intestine (Li) parts of the hindgut is drawn to scale before (stage 14) and after (stage 16) lumen diameter expansion. The hindgut lumen in thc mutants remains narrow. Bottom, left: Tnc (green) fills the lumen of the hindgut and appears as a striated matrix. Bottom, right: Over-expression of Tnc in the hindgut causes excess lumen dilation, both of inner (Crb; magenta, solid white line) and outer (Dystroglycan; blue, dashed white line) diameter, compared to the wild type. 


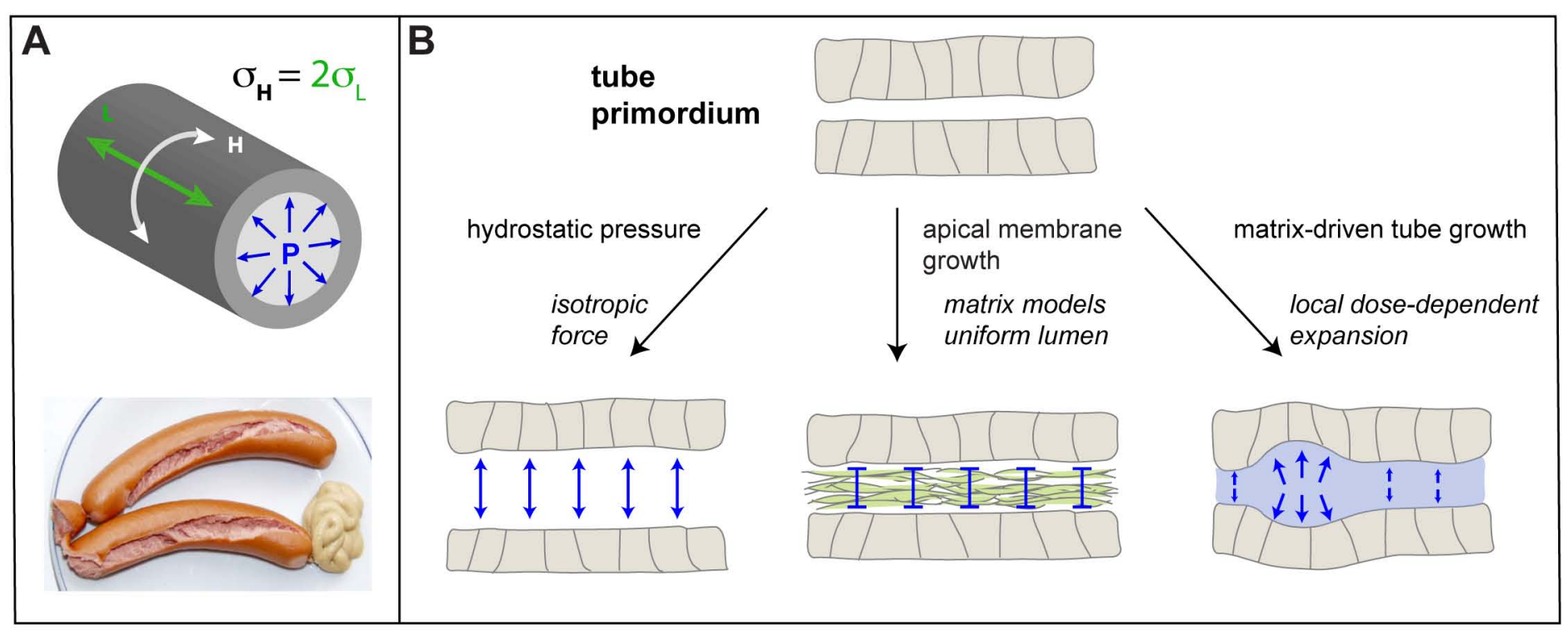




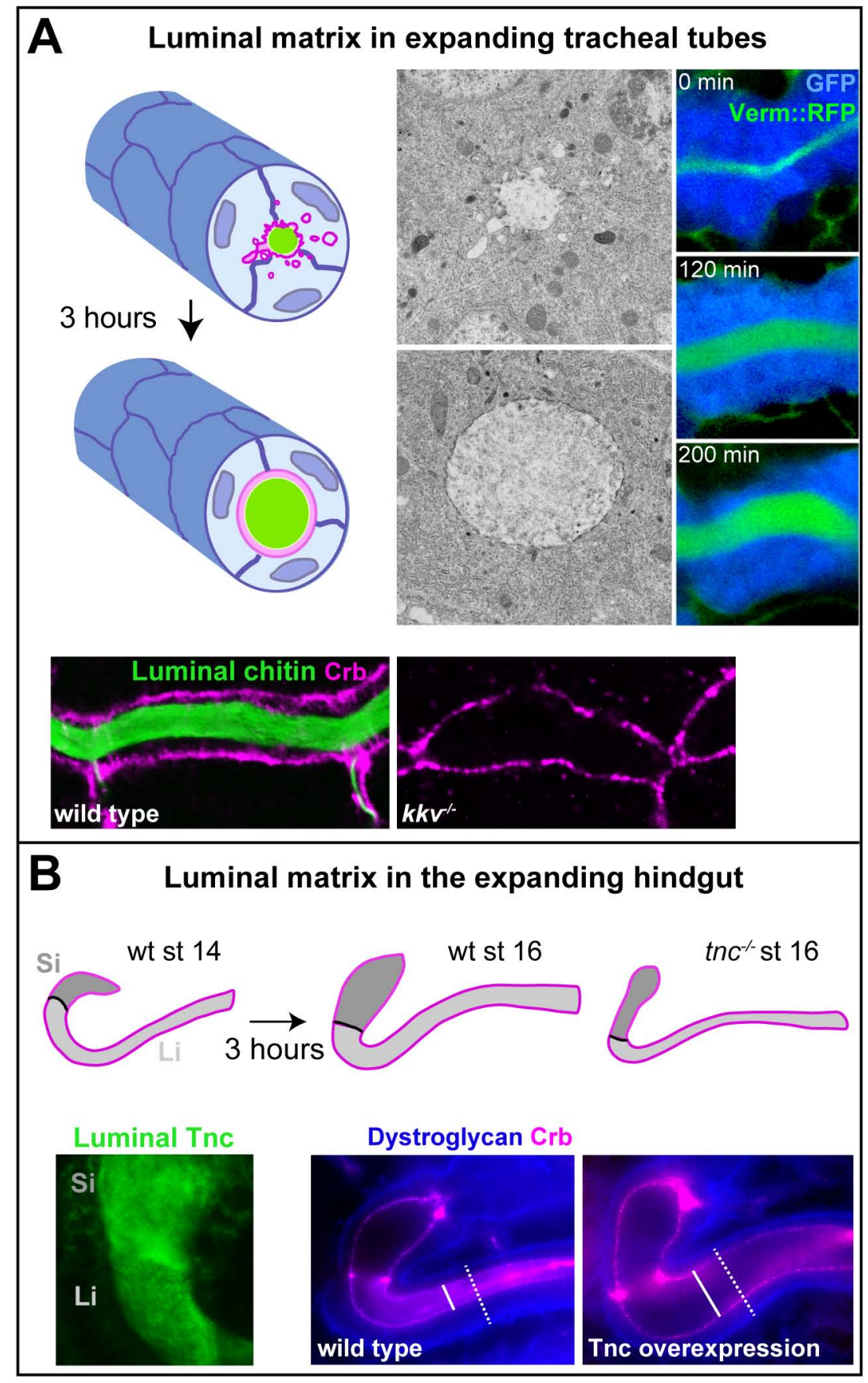

